\title{
Measurement of electromagnetic energy flow through a sparse particulate medium: A perspective
}

\author{
Michael I. Mishchenko* \\ NASA Goddard Institute for Space Studies, 2880 Broadway, New York, NY 10025, USA
}

\section{A R T I C L E I N F O}

\section{Article history:}

Received 24 November 2012

Received in revised form

26 December 2012

Accepted 7 January 2013

Available online 16 January 2013

Keywords:

Electromagnetic scattering

Directional radiometry

Radiative transfer

Optical particle characterization

Remote sensing

Macroscopic Maxwell equations

\begin{abstract}
A B S T R A C T
First-principle analysis of the functional design of a well-collimated radiometer (WCR) reveals that in general, this instrument does not record the instantaneous directional flow of electromagnetic energy. Only in special cases can a sequence of measurements with a WCR yield the magnitude and direction of the local time-averaged Poynting vector. Our analysis demonstrates that it is imperative to clearly formulate the physical nature of the actual measurement afforded by a directional radiometer rather than presume desirable measurement capabilities. Only then can the directional radiometer be considered a legitimate part of physically based remote sensing and radiation-budget applications. We also emphasize the need for a better understanding of the nature of measurements with panoramic radiometers.
\end{abstract}

Published by Elsevier Ltd.

\section{Introduction}

Measurements and computations of electromagnetic energy flow through a sparse particulate medium are traditionally viewed as an integral part of solving various radiation-budget and optical-characterization problems. For example, the physical state of a cloud of water droplets or ice crystals in the terrestrial atmosphere can be affected by an imbalance between the incoming and outgoing electromagnetic energy, while measurements of specific manifestations of electromagnetic energy flow with a suitable device can potentially be analyzed to infer useful information about the cloud. Conceptually similar problems are encountered in many other areas of science and technology.

Let us consider, for example, an idealized liquid-water cloud illuminated by a plane electromagnetic wave or, more generally, a quasi-monochromatic parallel beam of

\footnotetext{
* Tel.: +1 212678 5590; fax: +1 2126785552

E-mail addresses: michael.i.mishchenko@nasa.gov, crmim2@gmail.com
}

light with infinite lateral extent (Fig. 1). Suppose that we need to evaluate the radiation budget of a macroscopic volume element of the cloud $\Delta V$ bounded by the spherical surface $\Delta S$. According to the Poynting theorem [1], the net average rate at which electromagnetic energy enters this volume element is given by the integral

$W_{\Delta S}=-\int_{\Delta S} \mathrm{~d}^{2} \mathbf{r}\langle\mathbf{S}(\mathbf{r}, t)\rangle \cdot \hat{\mathbf{n}}(\mathbf{r})$,

where $\mathbf{S}(\mathbf{r}, t)$ is the Poynting vector at the point $\mathbf{r}$ at the moment $t$, the angular brackets denote averaging over a sufficiently long period of time, and the unit vector $\hat{\mathbf{n}}(\mathbf{r})$ is directed along the local outward normal to the boundary. If $W_{\Delta S}=0$ then the incoming radiation is balanced by the outgoing radiation. Otherwise there is absorption of electromagnetic energy inside the volume element. The radiation budget of the volume $V$ occupied by the entire cloud is evaluated similarly, except now the integral in Eq. (1) is taken over the closed boundary $S$ (Fig. 1 ).

Suppose that we have at our disposal a Poyntingmeter, i.e., a device that can measure both the direction and the absolute value of the time-averaged local Poynting vector. Then measuring $\langle\mathbf{S}(\mathbf{r}, t)\rangle$ at a sufficiently 


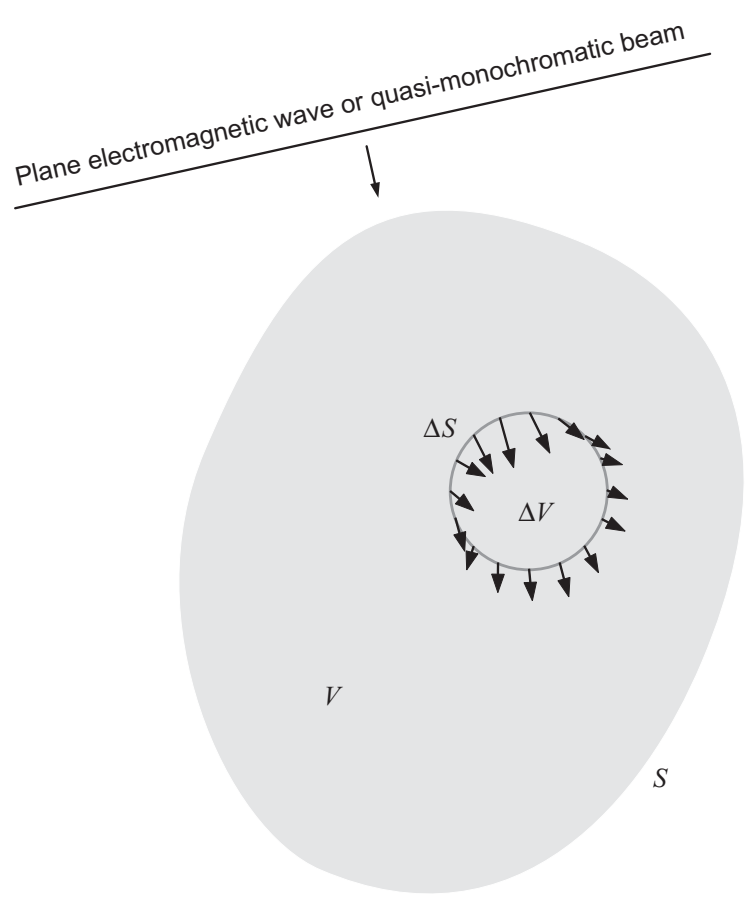

Fig. 1. Time-averaged radiation budget of a volume element $\Delta V$ of a cloud bounded by a closed surface $\Delta S$. The arrows represent the distribution of $\langle\mathbf{S}(\mathbf{r}, t)\rangle$ over the boundary $\Delta S$.

representative number of points densely distributed over the boundary $\Delta S$ and evaluating the integral in Eq. (1) numerically would solve the above radiation-budget problem.

Unfortunately, none of the instruments that have ever been used in the disciplines of atmospheric radiation and remote sensing can, strictly speaking, be considered a Poynting-meter. Despite the wide variety of specific designs and the alleged ability to quantify the electromagnetic energy flow [2], the actual physical nature of the measurements afforded by these instruments has remained poorly understood and has rarely been formulated in the context of advanced theories of light-matter interactions. Furthermore, it is hardly recognized that the physical meaning of the signal generated by these instruments depends critically on the very nature of the electromagnetic field transporting radiative energy and hence on the object creating the electromagnetic field.

Applied radiometry still appears to be dominated by the two-and-a-half century old phenomenology introduced by Pierre Bouguer and Johann Lambert [3,4], whereas very little has been done by way of clarifying the electromagnetic content of heuristic photometric concepts and putting measurements of electromagnetic energy flow in the context of modern physics. Even the most recent monographs on this subject (e.g., Ref. [2] and references therein) fall quite short of achieving this objective and can be thoroughly misleading in their usage of terminology borrowed in an ad hoc fashion from classical electromagnetism and quantum electrodynamics (QED).

In a series of reviews [5-7], I have attempted to summarize recent progress in the clarification of the fundamental physical content of electromagnetic scattering by particles and particulate media. Among other subjects, those reviews have focused on the precise nature of the phenomenon of electromagnetic scattering, the purely mathematical notions of multiple scattering and specific intensity in the framework of classical electromagnetics, and the derivation of the theories of radiative transfer and coherent backscattering directly from the macroscopic Maxwell equations. They have also dispelled the so-called "photonic confusion" in the context of phenomenological radiative transfer as well as the misconception of multidirectional propagation of electromagnetic energy allegedly described by the heuristic specific intensity.

This sequel is closely related to Refs. [5-7]. Its main objective is to clarify the physical framework of the measurement with instruments that can be called "wellcollimated radiometers" (WCRs). They represent by far the most widely used class of photometers, which makes it imperative to have a clear understanding of what these instruments can really measure and how their measurements are related to the requisite measurement of the Poynting vector. In particular, the following sections are intended to

- Summarize the basic operational principle of photoelectric detectors in the context of the QED theory of the photoelectric effect;

- Clarify the actual role of the optical tract of a WCR;

- Analyze the interaction of the electromagnetic radiation filtered out by the optical tract of a WCR with the end photodetector;

- Identify quantitative attributes of the electromagnetic energy flow that can be captured by a WCR depending on the specific measurement setting; and

- Discuss how these attributes can be modeled theoretically for morphologically complex scattering objects and thereby enter the solution of radiation-budget as well as optical-characterization problems.

\section{Photoelectric detectors}

Strictly speaking, the Poynting vector does not characterize the direction and rate of the local electromagnetic energy flow. Indeed, adding the curl of any vector field to $\mathbf{S}(\mathbf{r}, t)=\mathbf{E}(\mathbf{r}, t) \times \mathbf{H}(\mathbf{r}, t)$ yields a vector field $\mathbf{S}^{\prime}(\mathbf{r}, t)$ which also satisfies the Poynting theorem for the same pair of the electric and magnetic fields $\{\mathbf{E}(\mathbf{r}, t), \mathbf{H}(\mathbf{r}, t)\}$ $[1,8]$. It is, however, important to recognize that if we can measure the (time-averaged) Poynting vector then we can use Eq. (1) to evaluate the radiative energy budget of the object in question irrespective of the physical meaning of the vector product $\mathbf{E}(\mathbf{r}, t) \times \mathbf{H}(\mathbf{r}, t)$. Therefore, it is the practical measurability of the Poynting vector that will be the focus of the following discussion.

Typical devices used for the detection and quantification of electromagnetic energy flow are photomultipliers, photodiods, and photoelectric charge-coupled devices (CCDs) illustrated in Fig. 2. A photomultiplier or a 

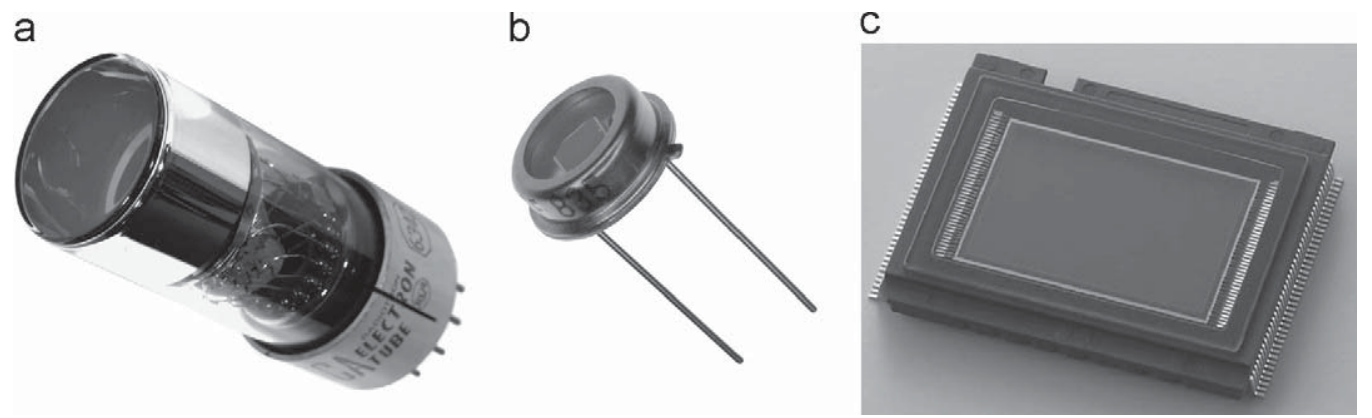

Fig. 2. Typical detectors of electromagnetic energy flow based on the photoelectric effect: (a) a photomultiplier, (b) a photodiod, and (c) a CCD.

photodiod is a single photodetector reacting to the electromagnetic field "impinging" anywhere on its relatively large sensitive surface, whereas a photoelectric CCD is a two-dimensional array of smaller detectors (pixels) which are designed to react to the incoming electromagnetic field independently of each other. All these devices are based on the absorption of electromagnetic energy via the photoelectric effect discovered by Heinrich Hertz in 1887 [9]. The physical nature of this phenomenon is well understood, both in the framework of the semiclassical approach, wherein only the matter is quantized while the electromagnetic field is treated classically $[10,11]$, and in the framework of quantum electrodynamics, wherein one quantizes both the matter and the electromagnetic field [11].

The essential property of the photoelectric effect is the Stoletov law according to which the photoelectric current is proportional to the intensity of the incident light. Although this law was discovered experimentally in 1889 [12], it has since been confirmed and refined theoretically. The principal result of the most general QED treatment today can be formulated as follows [13]. Let us approximate the sensitive element of a photodetector as a thin plane-parallel layer. The outer flat surface of this layer $S_{\mathrm{pd}}$ is exposed to a parallel beam of light which has an effectively infinite lateral extent, propagates normally to the surface, and consists of $N \geq 1$ quasimonochromatic components with different angular frequencies $\omega_{n}$, where $n$ numbers the quasi-monochromatic beams, so that $1 \leq n \leq N$. The local sensitivity of the detector is assumed to be uniform over $S_{\mathrm{pd}}$ and axially symmetric with respect to the local normal to $S_{\mathrm{pd}}$. Then the total number of photoelectrons recorded by the photodetector during a sufficiently long time interval $\Delta t$ is given by

$N_{\mathrm{pe}}(\Delta t)=\Delta t S_{\mathrm{pd}} \sum_{n=1}^{N} K^{\mathrm{pd}}\left(\omega_{n}\right)\left\langle I_{n}(t)\right\rangle$,

where $\left\langle I_{n}(t)\right\rangle$ is the time-averaged intensity of the $n$th quasi-monochromatic component and $K^{\mathrm{pd}}\left(\omega_{n}\right)$ is the quantum efficiency of the photodetector at the frequency $\omega_{n}$.

Despite the fundamental importance of Eq. (2), it is imperative to always remember the specific conditions of its applicability formulated in the preceding paragraph.

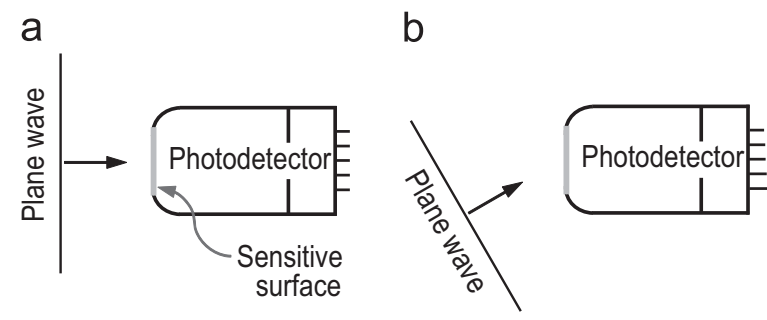

Fig. 3. A photodetector exposed to a plane electromagnetic wave.

Any violation of these conditions leads to complications and potentially significant uncertainties. Indeed, the angular distribution of the emitted photoelectrons is not isotropic and, furthermore, can depend on the polarization state of the incident beam $[14,15]$. The efficiency with which the photodetector collects the emitted photoelectrons propagating in various directions can never be perfectly uniform and, in reality, can be substantially anisotropic. This anisotropy can be expected to be especially pronounced for thicker sensitive layers [16]. As a consequence, real photodetectors can exhibit strong sensitivity to the direction of propagation and polarization state of the incident beam [17,18]. The polarization sensitivity can often be mitigated by placing a depolarizer in front of the photodetector, but the angular sensitivity may be more difficult to compensate for. It is, therefore, essential to limit the practical use of photoelectric detectors to the case of parallel beam illumination in the direction normal to the detector sensitive surface.

It is important to remember that Eq. (2) was specifically derived for a parallel polychromatic incident beam with quasi-monochromatic components propagating in the same direction, which makes it inapplicable to the case of uncollimated incident light.

\section{Photoelectric detector as a Poynting-meter}

The simplest photometer is a "bare" photoelectric detector with no optical elements, except, perhaps, a depolarizer, placed in front of its sensitive surface. Let us first assume that the detector is exposed to a plane electromagnetic wave propagating perpendicularly to its sensitive surface, as shown schematically in Fig. 3a. At any 
point of the sensitive surface, the direction of the instantaneous local Poynting vector is normal to the surface. Furthermore, the magnitude of the Poynting vector is uniform across the sensitive surface. It is well known [19] that the time-averaged Poynting vector of a plane electromagnetic wave is given by

$\langle\mathbf{S}(\mathbf{r}, t)\rangle=\hat{\mathbf{n}} I$,

where the unit vector $\hat{\mathbf{n}}$ specifies the direction of wave propagation and $I$ is the intensity of the wave. Since the direction $\hat{\mathbf{n}}$ is assumed to be known, the measurement illustrated in Fig. 3a can be thought of as yielding the time-averaged Poynting vector of the plane wave:

$\langle\mathbf{S}(\mathbf{r}, t)\rangle=\frac{N_{\mathrm{pe}}(\Delta t)}{\Delta t S_{\mathrm{pd}} K^{\mathrm{pd}}(\omega)} \hat{\mathbf{n}}$,

where $\omega$ is the corresponding angular frequency.

More generally, let us assume that the detector is illuminated by a superposition of $N$ parallel quasimonochromatic beams propagating in the same direction $\hat{\mathbf{n}}$ and having angular frequencies close enough so that for any $1 \leq n \leq N$,

$K^{\mathrm{pd}}\left(\omega_{n}\right) \approx K^{\mathrm{pd}}$.

Then Eq. (2) implies that

$\langle\mathbf{S}(\mathbf{r}, t)\rangle=\frac{N_{\mathrm{pe}}(\Delta t)}{\Delta t S_{\mathrm{pd}} K^{\mathrm{pd}}} \hat{\mathbf{n}}$,

where

$\langle\mathbf{S}(\mathbf{r}, t)\rangle=\sum_{n=1}^{N}\left\langle\mathbf{S}_{n}(\mathbf{r}, t)\right\rangle$

is the time-averaged Poynting vector of the total beam and $\left\langle\mathbf{S}_{n}(\mathbf{r}, t)\right\rangle$ is that of the $n$th quasi-monochromatic component. Thus in the limited measurement setting, wherein the direction of the Poynting vector is constant and is assumed to be known, the photodetector can be said to serve as the Poynting-meter.

In practice, the sensitive surface of the photodetector may be polarization sensitive, in which case the reading of the detector is proportional to $\langle I\rangle+\alpha_{Q}\langle Q\rangle+\alpha_{U}\langle U\rangle+$ $\alpha_{V}\langle V\rangle$, where one or more of the proportionality factors $\alpha_{Q}, \alpha_{U}$, and $\alpha_{V}$ are non-zero and

$\langle\mathbf{I}\rangle=\left[\begin{array}{c}\langle I\rangle=|\langle\mathbf{S}\rangle| \\ \langle Q\rangle \\ \langle U\rangle \\ \langle V\rangle\end{array}\right]$

is the (time-averaged) Stokes column vector of the plane electromagnetic wave or the parallel beam of light. In order to make the intensity measurement depicted in Fig. 3a useful, one must ensure that $\alpha_{Q}, \alpha_{U}$, and $\alpha_{V}$ are much smaller (in the absolute value sense) than unity. As we have indicated previously, this can often be accomplished by placing a depolarizer in front of the photodetector.

The interpretation of the measurement with the photodetector becomes more problematic if the propagation direction of the plane electromagnetic wave or the parallel beam of light is arbitrary and is not known a priori (see Fig. 3b). In this case the electromagnetic power intercepted by the sensitive surface scales as $\cos \theta$, where $\theta$ is the (unknown) angle between the propagation direction and the normal to the sensitive surface. Furthermore, the proportionality coefficients can also be expected to have a complex dependence on $\theta$. As a consequence, the reading of the photodetector is now proportional to $\cos \theta\left[\alpha_{I}(\theta)\langle I\rangle+\alpha_{Q}(\theta)\langle Q\rangle+\alpha_{U}(\theta)\langle U\rangle+\alpha_{V}(\theta)\langle V\rangle\right]$,

where $\alpha_{I}(\theta)$ is not necessarily equal to unity and $\theta$ is unknown. These factors make problematic the use of the photodetector as a Poynting-meter even if a high-quality depolarizer is placed in front of the sensitive surface.

Even more problematic is the situation depicted in Fig. 4. Now the photodetector is placed inside a cloud composed of $M$ randomly positioned and randomly moving particles and, thus, is located in the near zone of the cloud. This implies that the electromagnetic radiation interacting with the sensitive surface is neither a locally unidirectional transverse electromagnetic wave nor a superposition of quasi-monochromatic parallel beams propagating in the same direction. In other words, the radiation interacting with the sensitive surface is thoroughly uncollimated, which makes Eq. (2) inapplicable and the measurement afforded by the photodetector useless.

Even more fundamentally, the local Poynting vector at any point $\mathbf{r}$ of the sensitive surface at any moment in time is not the same as it would be in the absence of the detector. Indeed, let us assume for simplicity that the particles are separated widely enough to satisfy the conditions of applicability of the far-field Foldy-Lax equations (see Chapter 4 of Ref. [20]). Then the total instantaneous electric and magnetic fields at $\mathbf{r}$ in the absence of the detector are superpositions of the respective incident and $M$ scattered fields:

$\mathbf{E}(\mathbf{r}, t)=\mathbf{E}^{\mathrm{inc}}(\mathbf{r}, t)+\sum_{i=1}^{M} \mathbf{E}_{i}^{\text {sca }}(\mathbf{r}, t)$

$\mathbf{H}(\mathbf{r}, t)=\mathbf{H}^{\mathrm{inc}}(\mathbf{r}, t)+\sum_{i=1}^{M} \mathbf{H}_{i}^{\mathrm{sca}}(\mathbf{r}, t)$

where $\mathbf{E}^{\text {inc }}(\mathbf{r}, t)$ and $\mathbf{H}^{\text {inc }}(\mathbf{r}, t)$ represent the incident plane electromagnetic wave, while $\mathbf{E}_{i}^{\text {sca }}(\mathbf{r}, t)$ and $\mathbf{H}_{i}^{\text {sca }}(\mathbf{r}, t)$ describe an outgoing spherical wavelet centered at the origin of particle $i$. The corresponding local instantaneous Poynting

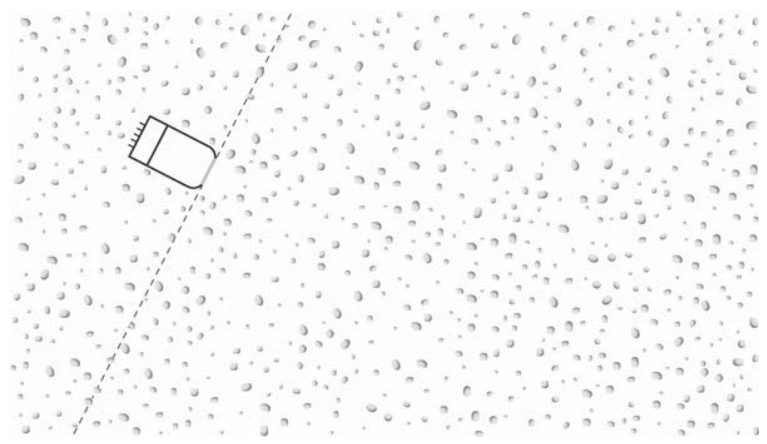

Fig. 4. A photodetector placed inside the scattering object in the form of a cloud of $M$ discrete particles. 
vector is given by the vector product $\mathbf{S}(\mathbf{r}, t)=\mathbf{E}(\mathbf{r}, t) \times \mathbf{H}(\mathbf{r}, t)$. The major side effect of the presence of the photodetector is to block the spherical wavelets generated by the $M^{\prime}$ particles located to the left of the plane through the sensitive surface shown schematically by the dashed line in Fig. 4. The resulting "truncated" electric and magnetic fields at $\mathbf{r}$ are now given by

$\mathbf{E}^{\prime}(\mathbf{r}, t)=\mathbf{E}^{\mathrm{inc}}(\mathbf{r}, t)+\sum_{i=1}^{M-M^{\prime}} \mathbf{E}_{i}^{\mathrm{sca}}(\mathbf{r}, t)$,
$\mathbf{H}^{\prime}(\mathbf{r}, t)=\mathbf{H}^{\mathrm{inc}}(\mathbf{r}, t)+\sum_{i=1}^{M-M^{\prime}} \mathbf{H}_{i}^{\mathrm{sca}}(\mathbf{r}, t)$,

respectively, where the sums include only the contributions from the $M-M^{\prime}$ unblocked particles. It is quite obvious that the corresponding "truncated" Poynting vector is not equal to the original Poynting vector: $\mathbf{S}^{\prime}(\mathbf{r}, t)=$ $\mathbf{E}^{\prime}(\mathbf{r}, t) \times \mathbf{H}^{\prime}(\mathbf{r}, t) \neq \mathbf{S}(\mathbf{r}, t)$.

In summary, the photodetectors shown in Fig. 2 can be called direction-insensitive owing to their inability to accurately decouple the direction and magnitude of the Poynting vector. The applicability of such instruments to the measurement of the Poynting vector is quite limited and relies on the following assumptions:

- the incoming radiation is a plane electromagnetic wave or a parallel polychromatic beam of light known to propagate perpendicularly to the sensitive surface of the photodetector;

- the incoming radiation is (or is made) unpolarized; and

- the range of angular frequencies of the incoming polychromatic beam is narrow enough that the quantum efficiency of the photodetector can be considered frequency-independent.

\section{Well-collimated radiometers}

A hypothetical instrument capable of measuring both the direction and the magnitude of the Poynting vector of a plane electromagnetic wave (or a parallel beam of light) is shown schematically in Fig. 5a. The sensitive surface of this instrument $S_{\mathrm{d}}$ is polarization-insensitive, is exposed directly to the incoming radiation, and is assumed to react to the local instantaneous Poynting vector only if this vector is directed along or almost along the optical axis of the instrument defined by the unit vector $\hat{\mathbf{q}}$ normal to $S_{\mathrm{d}}$. Specifically, if the direction of $\mathbf{S}\left(\mathbf{r}^{\prime}, t\right)$ at any point $\mathbf{r}^{\prime}$ of the sensitive surface at any moment $t$ is within the small acceptance solid angle $\Delta \Omega_{\hat{\mathbf{q}}}$ of the detector (e.g., vector $\mathbf{S}_{1}$ in Fig. 5a) then this vector contributes to the cumulative reading of the instrument. Instantaneous local Poynting vectors with directions outside $\Delta \Omega_{\hat{\mathbf{q}}}$ (e.g., vectors $\mathbf{S}_{2}$ and $\boldsymbol{S}_{3}$ ) are ignored by the instrument and do not contribute to its reading. The local time-averaged Poynting vector at the observation point $\mathbf{r}$ is then determined by (i) taking the time-averaged reading of the instrument $N_{\mathrm{pe}}(\mathbf{r}, \hat{\mathbf{q}}, \Delta t) / \Delta t$ at a dense grid of directions $\hat{\mathbf{q}} \in 4 \pi$ while keeping the sensitive surface centered at $\mathbf{r}$ and (ii) evaluating numerically the integral on the right-hand side of the following formula:

$$
\langle\mathbf{S}(\mathbf{r}, t)\rangle=\frac{1}{S_{\mathrm{d}} \Delta \Omega_{\hat{\mathbf{q}}} \Delta t K^{\mathrm{pd}}} \int_{4 \pi} \mathrm{d} \hat{\mathbf{q}} N_{\mathrm{pe}}(\mathbf{r}, \hat{\mathbf{q}}, \Delta t) \hat{\mathbf{q}} .
$$

The quantum efficiency of the detector is assumed to be frequency-independent.

It is obvious that this procedure could be expected to work in the case of collimated illumination, as illustrated in Fig. 6. However, the practical implementation of this measurement methodology faces two fundamental obstacles. First of all, to the best of the author's knowledge, the directional detector depicted schematically in Fig. 5a has never been built, and it remains quite questionable whether it can be built in principle. Obviously, assessing the very feasibility of a detector with directional sensitivity to the local instantaneous Poynting vector requires an advanced quantum-mechanical analysis of light-matter interaction. It is possible that there exists a variant of the Heisenberg uncertainty principle prohibiting such a measurement in principle.

Second of all, as we have already pointed out, if such an instrument were embedded in a cloud of particles then it would block the spherical wavelets generated by the particles located to the left of the plane through the sensitive surface shown schematically by the dashed line in Fig. 7. As a consequence, the "truncated" instantaneous local Poynting vector would be fundamentally different from the actual Poynting vector in the absence of the detector.

The majority of actual directional radiometers in use today are WCRs implicitly based on the physical principle illustrated schematically in Fig. 5b. The main functional elements of a WCR are the objective and relay lenses, the diaphragm, and the photoelectric detector. Let us consider the reaction of a WCR to the electromagnetic field formed by superposing two plane waves propagating in directions $\hat{\mathbf{q}}$ and $\hat{\mathbf{q}}^{\prime}$, respectively. The objective lens acts as a linear optical transformer in that its effect on the total field is a superposition of its effects on each plane-wave component. Specifically, the well-known paraxial approximation (e.g., Section 5.1 of Ref. [21]) implies that the objective lens transforms both plane wavefronts into converging spherical wavefronts with their respective focal points located in the plane of the diaphragm. However, the ultimate fate of the two spherical wavefronts is different. The pink spherical wavefront passes freely through the pinhole, is converted back into a plane wavefront, and is relayed onto the sensitive surface of the photodetector, thereby contributing to the cumulative reading of the WCR. On the other hand, the blue spherical wavefront gets annihilated by the diaphragm and does not contribute to the reading of the photodetector.

Thus the combination \{objective lens, diaphragm s serves to "extract" from the total electromagnetic field its components in the form of plane (or near-plane) wavefronts propagating in directions very close to the optical axis of the instrument and falling within its small acceptance solid angle

$\Delta \Omega=\frac{\pi d^{2}}{4 f^{2}}$,

where $d$ is the diameter of the pinhole and $f$ is the focal length of the objective lens. The fundamental feature of the 
a

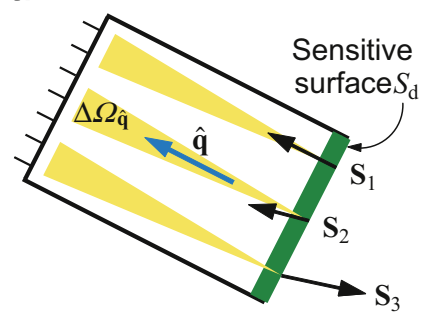

b

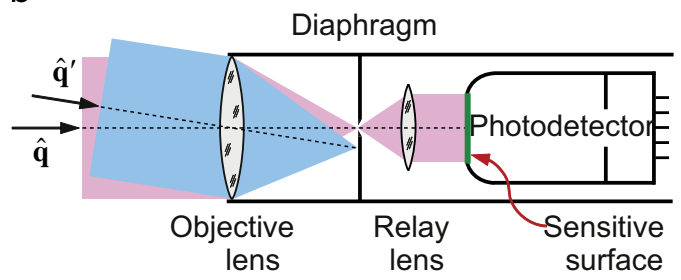

C

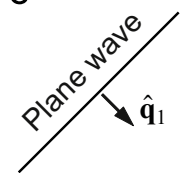

d

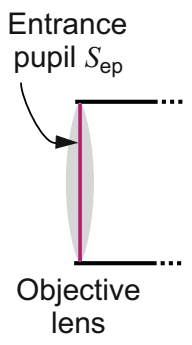

e

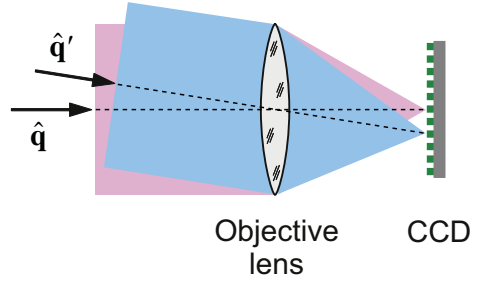

g

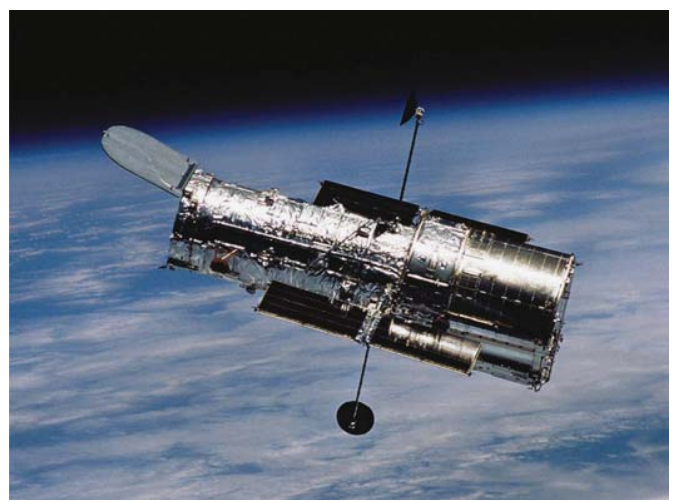

Fig. 5. (a) Hypothetical directional detector of electromagnetic energy. (b) Typical optical scheme of a well-collimated radiometer. (c) The conventional WCR does not respond to the Poynting vector directed along the optical axis of the instrument. (d) Entrance pupil of a WCR. (e) Panoramic radiometer. (f) 6-m telescope of the Russian Academy of Sciences. (g) NASA's Hubble Space Telescope.

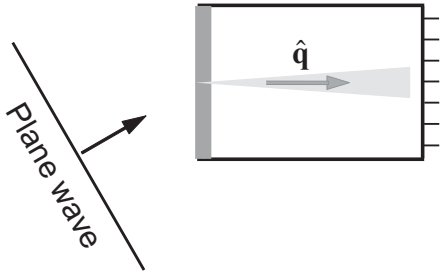

Fig. 6. A hypothetical directional detector exposed to a plane electromagnetic wave.

instrument shown in Fig. 5b is that it selects appropriately directed wavefronts rather than appropriately directed instantaneous Poynting vectors [22]. It can thus be said that a WCR acts as a wave-domain filter rather than a Poyntingmeter.

An important implication of this analysis is that the typical WCR does not necessarily react to the local Poynting

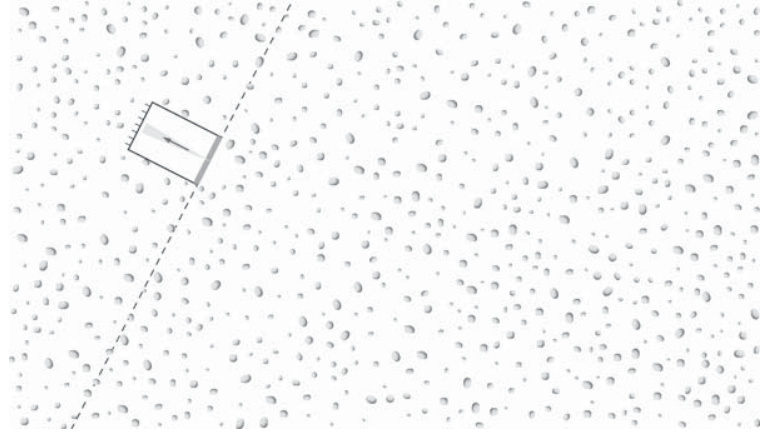

Fig. 7. A hypothetical directional detector placed inside a cloud of particles.

vector at a point on the exterior surface of the objective lens even if this vector is directed along the optical axis of the instrument [22]. To demonstrate this, let us consider two 
plane electromagnetic waves propagating in directions $\hat{\mathbf{q}}_{1}$ and $\hat{\mathbf{q}}_{2}$ such that both form a $45^{\circ}$ angle with the optical axis of the WCR (Fig. 5c). Let us assume that the waves are linearly polarized, with their electric vectors $\mathbf{E}_{1}$ and $\mathbf{E}_{2}$ oscillating perpendicularly to the paper, and fully coherent in that at any moment in time $\mathbf{E}_{1}=\mathbf{E}_{2}$ at the central point of the objective lens. Let the local instantaneous magnetic vectors of the waves be $\mathbf{H}_{1}$ and $\mathbf{H}_{2}$, respectively, as shown by the magenta arrows, while the corresponding instantaneous electric vectors $\mathbf{E}_{1}=\mathbf{E}_{2}$ are directed towards the reader. The total local instantaneous field is represented by the vectors $\mathbf{E}=2 \mathbf{E}_{1}$ and $\mathbf{H}=\mathbf{H}_{1}+\mathbf{H}_{2}$, the former again being directed towards the reader. One can see that the resulting local instantaneous Poynting vector $\mathbf{S}=\mathbf{E} \times \mathbf{H}$, shown by the green arrow, is directed along the optical axis of the WCR. Furthermore, it is easily verified that the Poynting vector at the central point is always directed along the optical axis of the instrument. Yet the reading of the photodetector is identically equal to zero since neither plane wavefront is passed by the \{objective lens, diaphragm\} filter of the WCR.

Conversely, a WCR can generate a nonzero signal when the local Poynting vector is zero. Indeed, let us consider the superposition of two plane waves propagating in opposite directions along the $y$-axis, as shown in Fig. 8. The corresponding electric and magnetic fields are given by

$\mathbf{E}_{1}(\mathbf{r}, t)=E \cos (k y-\omega t) \hat{\mathbf{z}}, \quad \mathbf{H}_{1}(\mathbf{r}, t)=H \cos (k y-\omega t) \hat{\mathbf{X}}$,

$\mathbf{E}_{2}(\mathbf{r}, t)=E \cos (k y-\omega t) \hat{\mathbf{z}}, \quad \mathbf{H}_{2}(\mathbf{r}, t)=-H \cos (k y-\omega t) \hat{\mathbf{x}}$,

respectively, where $\hat{\mathbf{x}}$ and $\hat{\mathbf{z}}$ are unit Cartesian vectors. One can see that at the origin $(y=0)$, the total magnetic field and, as a consequence, the Poynting vector are identically equal to zero. Yet the WCRs in Fig. $8 \mathrm{~b}$ and c block one of the waves and thereby record a nonzero "Poynting vector" directed along the positive (Fig. 8b) or negative (Fig. 8c) $y$-axis.

The failure of the WCR to react to the instantaneous Poynting vector in Fig. $5 \mathrm{c}$ can be traced to the following fundamental fact: although the Poynting vector is implicitly sought at points on the exterior surface of the objective lens, the actual photodetector is invariably located very far (compared to the wavelength) from these points. What the optical scheme of the WCR can relay from the entrance plane onto the sensitive surface of the photodetector is a suitable plane (or quasi-plane) wavefront, but not the Poynting vector of the total field. The only circumstance in which the WCR relays the Poynting vector itself is when the total field consists only of one or several plane or near-plane wavefronts propagating in the same direction along the optical axis of the instrument.

This is true of any WCR irrespective of its specific optical scheme. In fact, it is the very principle of serving as a wavefront angular filter (rather than a Poynting vector angular filter) that allows one to build a WCR by using easy-to-fabricate macroscopic optical elements (such as lenses, mirrors, polarizers, prisms, diffraction gratings, etc.). In some cases these elements can be very large, as exemplified by modern astronomical telescopes (Fig. 5f,g).

Thus, contrary to a widespread misconception, a WCR cannot be said to measure the directional distribution of the electromagnetic energy flow at an observation point. It is therefore imperative to formulate precisely what a WCR does
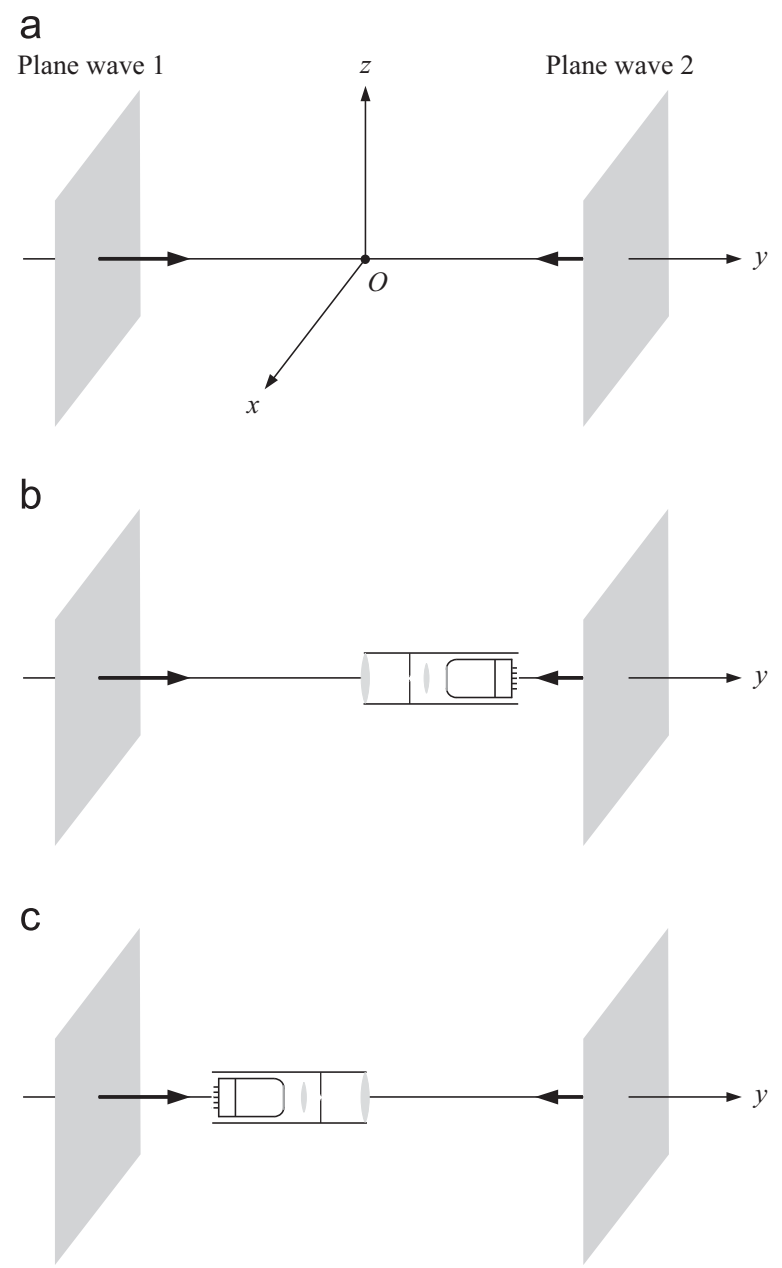

Fig. 8. A WCR can register a nonzero signal even if the local Pointing vector is identically equal to zero.

in actuality. Let us assume that it is exposed to an electromagnetic field in the form of a superposition of several plane wavefronts, as depicted schematically in Fig. 9. According to the above discussion, the WCR does the following:

- selects only the wavefronts with propagation directions falling within its acceptance solid angle $\Delta \Omega_{\hat{\mathbf{q}}}$ (i.e., $\hat{\mathbf{q}}_{3}, \hat{\mathbf{q}}_{4}$, and $\hat{\mathbf{q}}_{5}$, but not $\hat{\mathbf{q}}_{1}, \hat{\mathbf{q}}_{2}, \hat{\mathbf{q}}_{6}$, and $\hat{\mathbf{q}}_{7}$ );

- sums up the respective instantaneous electric and magnetic fields: $\mathbf{E}^{\prime}=\mathbf{E}_{3}+\mathbf{E}_{4}+\mathbf{E}_{5}$ and $\mathbf{H}^{\prime}=\mathbf{H}_{3}+\mathbf{H}_{4}+\mathbf{H}_{5}$; and finally

- integrates the vector product $\mathbf{E}^{\prime} \times \mathbf{H}^{\prime}$ (which, by its very construct, is always directed along-or very close to-the optical axis of the WCR) over the entrance pupil $S_{\text {ep }}$ as well as over time.

Importantly, invoking the concept of the flat entrance pupil of a WCR, shown in Fig. 5d, helps to accommodate the following two facts:

- the electromagnetic power enters the WCR through the objective lens and is, therefore, proportional to the area of this lens; and 


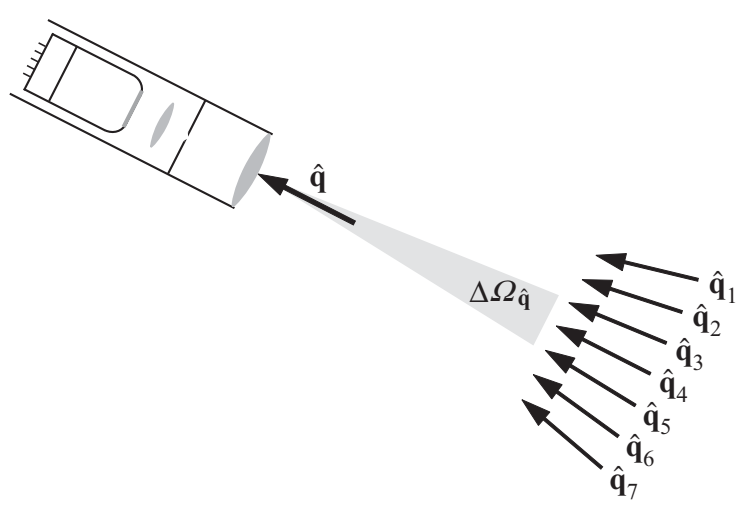

Fig. 9. Response of a WCR to the electromagnetic field formed by a superposition of several plane electromagnetic waves.

- the actual registration of the resulting Poynting vector of the superposition of transmitted wavefronts occurs at the sensitive surface of the photodetector.

Indeed, the optical scheme of the WCR serves to preserve the phase relations between the incoming wavefronts as they are relayed onto the sensitive surface of the detector. Suppose, for example, that one of two wavefronts propagating along the WCR axis is a plane electromagnetic wave $\left\{\mathbf{E}_{1}, \mathbf{H}_{1}\right\}$ while the other one is a spherical wave $\left\{\mathbf{E}_{2}\right.$, $\mathbf{H}_{2}$ \} with a large but finite radius of curvature. The phase difference between these wavefronts varies over a plane perpendicular to the direction of propagation, thereby causing lateral variations of the resulting vector product $\left(\mathbf{E}_{1}+\mathbf{E}_{2}\right) \times\left(\mathbf{H}_{1}+\mathbf{H}_{2}\right)$. It is easy to see that the combination of the objective and relay lenses in Fig. 5b yields the same phase difference distribution over the sensitive surface of the detector as that over the flat entrance pupil. We will see in Section 5 that this functional feature of a WCR is essential in the practical measurement of extinction.

Another fundamental feature of the basic optical scheme of a WCR is that the wavefronts impinging on the sensitive surface of the photodetector always propagate normally to the surface, thereby eliminating one of the drawbacks of uncollimated radiometers discussed in Section 3 and making applicable Eq. (2). Although the photodetector can still have residual polarimetric sensitivity, this problem can be mitigated by inserting a depolarizer between the relay lens and the detector.

Thus, the operation performed by a WCR is by no means equivalent to accumulating appropriately directed local instantaneous Poynting vectors of the total field over the entrance pupil (cf. Fig. 5a). Despite this fundamental limitation, the above discussion shows that the measurement afforded by a WCR is well defined and thus can be modeled theoretically. This, as will be discussed below, makes WCRs very useful tools in solving radiation-budget and particle characterization problems. Furthermore, a WCR can be upgraded to measure even the state of polarization of the superposition of wavefronts filtered out by its \{objective lens, diaphragm combination (see, e.g., Section 2.10 of Ref. [20]). This further increases the utility of WCRs in optical particle characterization.
The very fact that a WCR serves as a directional filter of plane or near-plane wavefronts imposes strict limitations on its practical use. For example, a WCR having a centimetersized or larger entrance pupil cannot be used to characterize the near field created by a micrometer-sized particle. Solving this problem requires specifically designed nanooptical probes [23] and is beyond the scope of this paper. Instead, as will be discussed in the following section, a WCR should ideally be located in the far zone of the entire scattering object and at a distance from the object much greater than the diameter of the entrance pupil. Then the part of the scattered spherical wavefront cut out by the entrance pupil becomes sufficiently flat to pass in its entirety the \{objective lens, diaphragm \} filter.

The WCR shown in Fig. 10 is located in the near zone of the cloud. We have mentioned, however, that if the cloud is formed by widely separated particles then the total field at any observation point located in the far zones of all the constituent particles is a superposition of the incident field and individual spherical wavelets contributed by all the cloud particles. If the size of the cloud is much greater than the diameter of the entrance pupil then the majority of these wavelets are contributed by remote particles and have near-plane wavefronts at the location of the WCR. Therefore, the use of the WCR appears to be justified as long as these wavefronts dominate the total local field.

An implicit advantage of a WCR over the hypothetical directional detector shown in Fig. $5 \mathrm{a}$ is that the specific measurement afforded by the WCR is much less affected by the WCR's very presence. Indeed, we have seen before that placing the hypothetical directional detector inside a cloud of particles would change the instantaneous local Poynting vector dramatically by blocking a large fraction of incoming spherical wavefronts (Fig. 7). The measurement afforded by the WCR is fundamentally different in that, by definition, the WCR collects only those incoming spherical wavefronts that are generated by particles residing in the narrow conical volume $\Delta V_{\hat{\mathbf{q}}}$ defined by the WCR's acceptance solid angle $\Delta \Omega_{\hat{\mathbf{q}}}$ (Fig. 10). It is obvious that placing the WCR inside the cloud blocks none of these wavefronts.

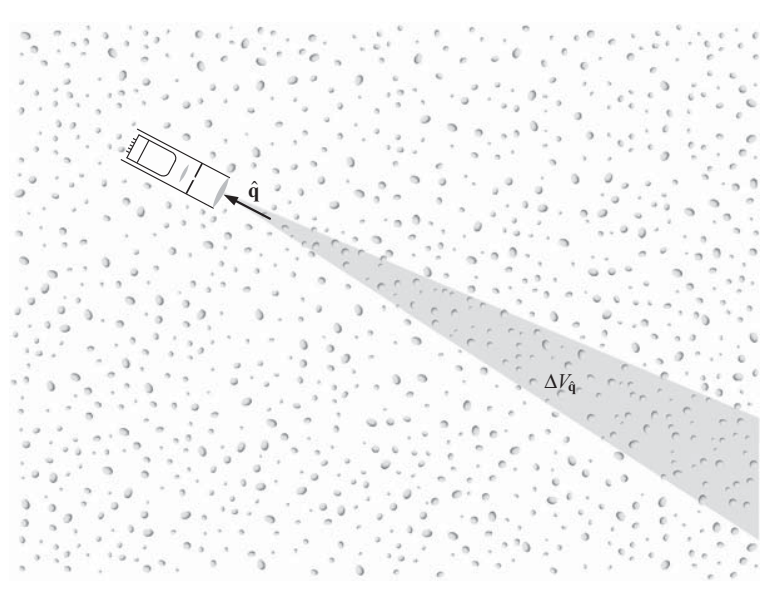

Fig. 10. A WCR placed inside a random cloud of particles. 
Admittedly, the addition of a foreign body such as a WCR modifies the partial wavelets created at the observation point by the particles residing in the conical volume $\Delta V_{\hat{\mathbf{q}}}$. However, the WCR can be expected to affect only the wavelets coming from particles located in a close vicinity of the WCR. If the size of the WCR is much smaller than that of the cloud then the fraction of such particles in the conical volume $\Delta V_{\hat{\mathbf{q}}}$ is relatively small, thereby making the actual signal measured by the WCR close to the imaginary signal caused by the same particles in the absence of the WCR. The situation depicted in Fig. 7 is fundamentally different in that the hypothetical directional detector essentially destroys the signal that it is supposed to measure.

\section{Far-field scattering}

Scattering in the far zone is unique in that the scattered field always evolves into a simple outgoing spherical wave irrespective of the physical nature of the scatterer (e.g., Section 3.2 of Ref. [20]). It should therefore be instructive to analyze how the main results of the preceding section apply to this simplest type of electromagnetic scattering. Specifically, the aim of this section is to describe far-field scattering in terms of quantities directly measurable with a WCR and/or directly quantifying the radiation energy budget of a finite volume element enclosing the scattering object.

Consider the measurement configuration involving polarization-insensitive WCRs located at a distance $r$ from the origin $O$ in the far zone of the entire scattering object, as shown in Fig. 11. The origin of the laboratory coordinate system is centered at the object. The total electromagnetic field at the location of a WCR is mathematically represented as a superposition of the incident field in the form of a plane electromagnetic wave propagating in the direction of the unit vector $\hat{\mathbf{n}}^{\text {inc }}$ and the scattered field in

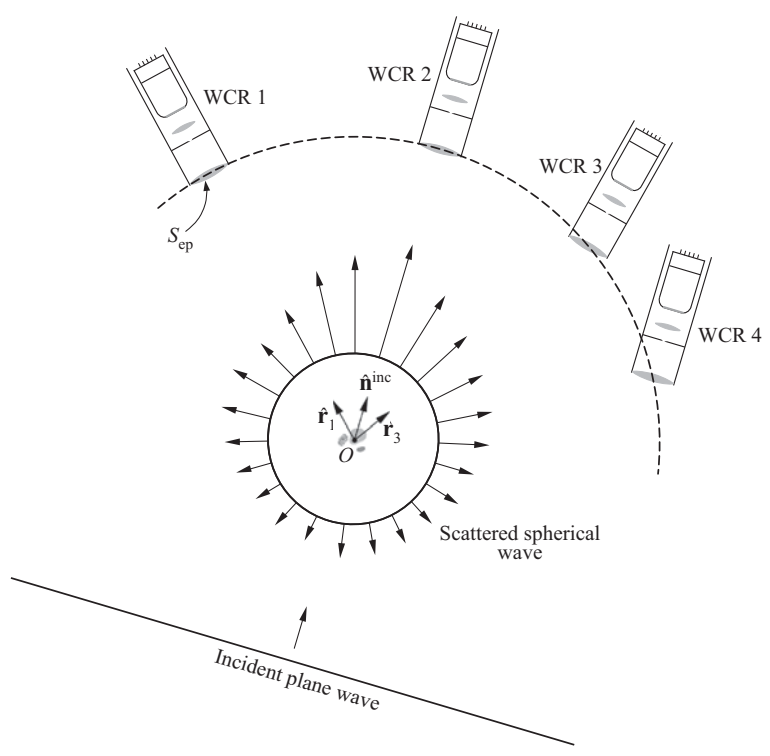

Fig. 11. Electromagnetic response of a WCR depends on the line of sight. the form of an outgoing spherical wave centered at 0 . It is important to recognize that the total field is the only real physical field, while the separation of this field into the incident and scattered components is notional only [5]. The mathematical relationship between the incident and scattered fields in the far zone is specified in terms of the $2 \times 2$ so-called amplitude scattering matrix [19,20].

The entrance pupils of the four WCRs shown in Fig. 11 are centered at the endpoints of the respective position vectors $\mathbf{r}_{1}=r \hat{\mathbf{r}}_{1}, \quad \mathbf{r}_{2}=r \hat{\mathbf{r}}_{2}=r \hat{\mathbf{n}}^{\text {inc }}, \quad \mathbf{r}_{3}=r \hat{\mathbf{r}}_{3}$, and $\mathbf{r}_{4}=r \hat{\mathbf{r}}_{4}$. The distance $r$ from the origin is assumed to be much greater than the diameter $D$ of the entrance pupils to ensure that the part of the scattered wavefront (shown by the dashed curve in Fig. 11) cut out by an entrance pupil is essentially flat. Specifically, it satisfies the inequality

$\frac{S_{\mathrm{ep}}}{r^{2}}<\Delta \Omega$,

where $S_{\mathrm{ep}}=\pi D^{2} / 4$ is the area of the entrance pupil and, as before, $\Delta \Omega$ is the WCR acceptance solid angle defined by Eq. (14).

The optical axis of WCR 3 coincides neither with the respective radial direction $\hat{\mathbf{r}}_{3}$ nor with the incidence direction $\hat{\mathbf{n}}^{\text {inc }}$. According to the discussion in Section 4, this implies that neither the incident plane-wave component nor the locally flat scattered-wave component can pass the \{objective lens, diaphragm filter, which makes the reading of WCR 3 identically equal to zero. In other words, if the optical axis of a WCR does not go through the origin $O$ and is not parallel to $\hat{\mathbf{n}}^{\text {inc }}$ then the WCR records no signal.

The optical axis of WCR 4 is parallel to the incidence direction but does not coincide with the respective radial direction $\hat{\mathbf{r}}_{4}$, which implies that only the incident planewave component passes the \{objective lens, diaphragm filter. Therefore, the time-averaged total electromagnetic power recorded by WCR 4 is given by

$\left\langle W_{4}\right\rangle=S_{\text {ep }} I^{\text {inc }}$,

where $I^{\text {inc }}$ is given by the intensity of an electromagnetic field represented by the incident plane-wave component only, i.e., as if the scattered spherical wave component were zero.

The optical axis of WCR 1 coincides with the corresponding radial direction $\hat{\mathbf{r}}_{1}$, but not with the incidence direction $\hat{\mathbf{n}}^{\text {inc }}$. Therefore, the \{objective lens, diaphragm filter of WCR 1 passes only the scattered wavefront, and the time-averaged total electromagnetic power recorded by WCR 1 is given by

$\left\langle W_{1}\left(\hat{\mathbf{r}}_{1}\right)\right\rangle=S_{\mathrm{ep}} I^{\mathrm{sca}}\left(r \hat{\mathbf{r}}_{1}\right)$,

where $I^{\text {sca }}\left(r \hat{\mathbf{r}}_{1}\right)$ is given by the intensity of an electromagnetic field represented by the scattered spherical wave component only, i.e., as if the incident plane-wave component were zero.

The computation of the electromagnetic response of WCR 2 oriented along the incidence direction is more involved because the corresponding \{objective lens, diaphragm filter passes both the incident and forward-scattered wavefronts. The integration of the corresponding time-averaged Poynting vector $\left\langle\mathbf{S}\left(\mathbf{r}^{\prime}, t\right)\right\rangle$ over all 
points $\mathbf{r}^{\prime}$ of the entrance pupil of WCR 2 ultimately yields the optical theorem $[19,20]$ :

$$
\left\langle W_{2}\right\rangle=S_{\mathrm{ep}} I^{\text {inc }}-C_{\mathrm{ext}} I^{\text {inc }} \text {, }
$$

where $C_{\text {ext }}$ is the extinction cross section. The first term on the right-hand side of Eq. (20) is equal to the electromagnetic power that would be recorded by WCR 2 in the absence of the scattering object. The second term is independent of $S_{\text {ep }}$ and describes attenuation caused by interposing the object between the light source and the WCR. Thus, the WCR centered at the object along the forwardscattering direction can be said to measure the power of the incident plane wave attenuated by the interference of the incident and scattered wavefronts. The detector centered at the object along any other radial direction reacts only to the scattered wavefront.

The fact that the extinction term in Eq. (20) is independent of $S_{\mathrm{ep}}$ has a simple qualitative explanation. Although the radius of curvature of the scattered wavefront at the location of WCR 2 is large, it is still finite. Because of that, the phase difference between the incident and scattered wavefronts varies over the flat entrance pupil (see Fig. 12), thereby causing the interference component of the cumulative Poynting vector to oscillate with increasing frequency as the distance of the observation point $\mathbf{r}^{\prime}$ from the center of the entrance pupil increases. These high-frequency oscillations between positive and negative values effectively cancel each other upon the integration over the entrance pupil. The only exception is the immediate vicinity of the exact forwardscattering direction, where the phase difference between the incident and scattered wavefronts remains

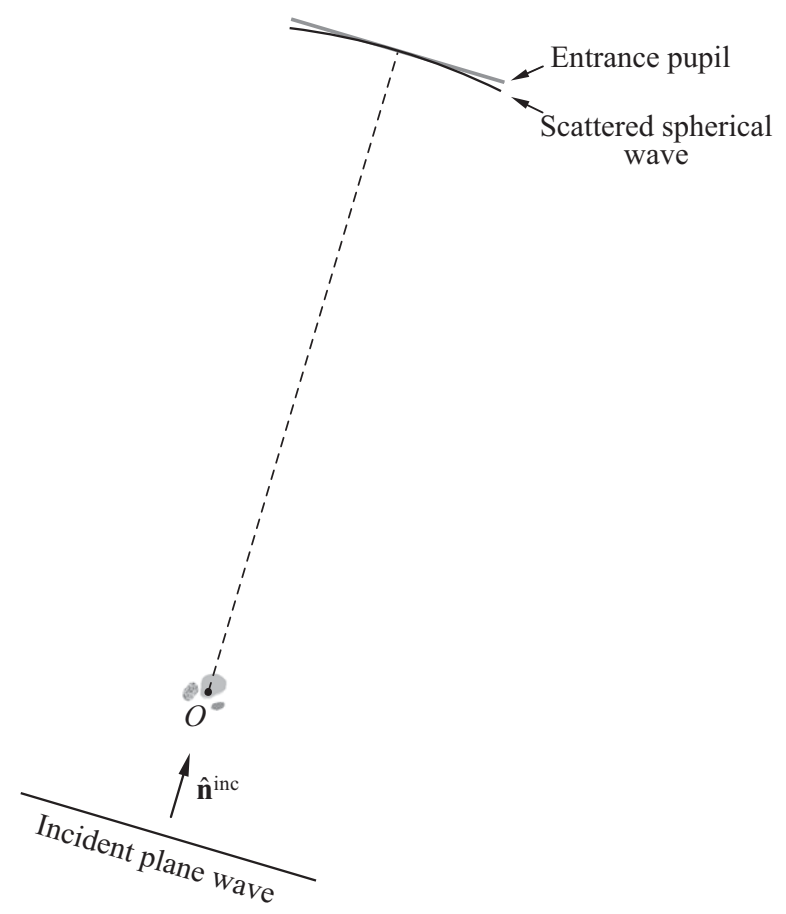

Fig. 12. The scattered spherical wavefront "interacts" with the incident plane wavefront only in the immediate vicinity of the exact forwardscattering direction. approximately constant, thereby causing a nonvanishing negative contribution to the signal measured by WCR 2 [24-27]. This explanation also demonstrates the importance of the optical scheme of the WCR preserving the lateral distribution of the phase difference between the incident and scattered wavefronts as they are relayed onto the sensitive surface of the photodetector (recall the discussion in Section 4).

Let us now go back to Fig. 11. It is easily seen that the Poynting vector of the total field $\mathbf{S}\left(\mathbf{r}^{\prime}, t\right)$ at any point $\mathbf{r}^{\prime}$ of the entrance pupil of WCR 1 is never directed along the optical axis of the instrument since the electric and magnetic field vectors $\mathbf{E}\left(\mathbf{r}^{\prime}, t\right)$ and $\mathbf{H}\left(\mathbf{r}^{\prime}, t\right)$ of the total field are never parallel to the pupil plane. Yet, WCR 1 records a nonzero timeaveraged signal described by Eq. (19). Similarly, the Poynting vector $\mathbf{S}\left(\mathbf{r}^{\prime}, t\right)$ at any point $\mathbf{r}^{\prime}$ of the entrance pupil of WCR 4 is never directed along the incidence direction, and yet WCR 4 registers a nonzero time-averaged signal given by Eq. (18). On the other hand, the Poynting vector $\mathbf{S}\left(\mathbf{r}^{\prime}, t\right)$ can be directed along the optical axis of WCR 3 at certain points of its entrance pupil and at certain moments in time. Despite that, WCR 3 records no signal whatsoever. These facts demonstrate again that in general, a WCR does not measure the directional flow of electromagnetic energy but rather generates a certain signal defined by its specific functional design. The only exception is the case when all the wavefronts constituting the local electromagnetic field are parallel to the entrance pupil of the WCR. Only WCR 2 in Fig. 11 satisfies this condition and hence integrates over its entrance pupil the actual time-averaged Poynting vector of the total electromagnetic field. Thus, Fig. 11 demonstrates that it is imperative indeed to understand the physical nature of the actual measurement afforded by a WCR rather than to ascribe to the WCR a desirable measurement capability.

The practical usefulness of Eqs. (19) and (20) comes from the fact that $I^{\text {sca }}(r \hat{\mathbf{n}})$ and $C_{\text {ext }}$ can be measured experimentally as well as calculated by using a computer solver of the macroscopic Maxwell equations. They thus constitute key ingredients of various optical techniques intended for non-invasive characterization of morphological and compositional properties of natural and artificial particulates. The versatility and accuracy of these techniques can be further enhanced by converting polarizationinsensitive WCRs into high-accuracy polarimeters and using appropriate "vector" generalizations of Eqs. (18)-(20) (see, e.g., Sections 3.7 and 3.8 of Ref. [20]).

Let us now assume that we want to measure the radiation budget of a spherical volume element $\Delta V$ surrounding the scattering object and centered at $O$. The radius $r$ of the spherical boundary $\Delta S$ in Eq. (1) is assumed to be sufficiently large for the boundary to reside in the far zone of the object. The application of the Poynting theorem (1) to the volume element $\Delta V$ yields

$W_{\Delta S}=-\int_{\Delta S} \mathrm{~d}^{2} \mathbf{r}\langle\mathbf{S}(\mathbf{r}, t)\rangle \cdot \hat{\mathbf{n}}(\mathbf{r})=-r^{2} \int_{4 \pi} \mathrm{d} \hat{\mathbf{n}} I^{\mathrm{sca}}(r \hat{\mathbf{n}})+C_{\mathrm{ext}} I^{\mathrm{inc}}$.

Comparison of Eqs. (18)-(20) with Eq. (21) shows that although WCRs 1 and 4 never react to the local timeaveraged Poynting vector of the total field, they can still be used to evaluate the radiation budget of the volume element $\Delta V$ experimentally. 
The results of this section can easily be generalized to the case of polychromatic radiation assuming, as before, that the quantum efficiency of the photodetectors remains constant over the entire spectral range involved.

\section{Near-field scattering}

Let us now discuss what happens when a WCR is placed inside a random cloud populated by $M$ sparsely distributed particles (Fig. 10). Again, the instantaneous real electric and magnetic vectors at an observation point $\mathbf{r}$ can be represented mathematically as superpositions of the respective vectors of the incident field and the partial fields "coming" from all the individual particles according to Eqs. (9) and (10), where each pair $\left\{\mathbf{E}_{i}(\mathbf{r}, t), \mathbf{H}_{i}(\mathbf{r}, t)\right\}$ represents an outgoing spherical wavelet originating at the center of the respective particle. At a large distance from the particle this wavelet can be considered locally flat. Therefore, the WCR will select only those wavelets that come from the particles located within the "acceptance volume" $\Delta V_{\hat{\mathbf{q}}}$ of the radiometer defined by its acceptance solid angle $\Delta \Omega_{\hat{\mathbf{q}}}$ (Fig. 10) and will integrate the corresponding composite Poynting vector over the entrance pupil $S_{\text {ep }}$. Assuming for simplicity that $\Delta \Omega_{\hat{\mathbf{q}}}$ does not subtend the propagation direction of the incident light $\hat{\mathbf{n}}^{\text {inc }}$, we conclude that the instantaneous value of the composite Poynting vector is given by

$\sum_{l^{\prime}} \sum_{m^{\prime}} \mathbf{E}_{l^{\prime}}(\mathbf{r}, t) \times \mathbf{H}_{m^{\prime}}(\mathbf{r}, t)$

where the primed indices $l^{\prime}$ and $m^{\prime}$ number particles positioned inside the acceptance volume $\Delta V_{\hat{\mathbf{q}}}$. Note that since $\Delta \Omega_{\hat{\mathbf{q}}}$ is very small, each term in the sum (22) is a vector directed essentially along the unit vector $\hat{\mathbf{q}}$ in Fig. 10. Averaging this reading over a sufficiently long time interval $\Delta t$ yields the following average signal per unit area of the entrance pupil:

$\left\langle\sum_{l^{\prime}} \sum_{m^{\prime}} \mathbf{E}_{l^{\prime}}(\mathbf{r}, t) \times \mathbf{H}_{m^{\prime}}(\mathbf{r}, t)\right\rangle$.

This quantity can be computed analytically using the standard assumptions of the microphysical theory of radiative transfer [20] such as ergodicity of the random $M$-particle ensemble, the limit $M \rightarrow \infty$, the Twersky approximation, and the ladder approximation. The result of this computation [22] implies that the reading of the WCR in Fig. 10 per unit time is given by the product

$S_{\mathrm{ep}} \Delta \Omega_{\hat{\mathbf{q}}} \tilde{I}(\mathbf{r}, \hat{\mathbf{q}})$,

where $\tilde{I}(\mathbf{r}, \hat{\mathbf{q}})$ is the first element of the four-element column $\tilde{\mathbf{I}}(\mathbf{r}, \hat{\mathbf{q}})$ satisfying the following integro-differential equation:

$\hat{\mathbf{q}} \cdot \nabla \tilde{\mathbf{I}}(\mathbf{r}, \hat{\mathbf{q}})=-n_{0}\langle\mathbf{K}(\hat{\mathbf{q}})\rangle_{\xi} \tilde{\mathbf{I}}(\mathbf{r}, \hat{\mathbf{q}})+n_{0} \int_{4 \pi} \mathrm{d} \hat{\mathbf{q}}^{\prime}\left\langle\mathbf{Z}\left(\hat{\mathbf{q}}, \hat{\mathbf{q}}^{\prime}\right)\right\rangle_{\xi} \tilde{\mathbf{I}}\left(\mathbf{r}, \hat{\mathbf{q}}^{\prime}\right)$.

Here, $n_{0}=N / V$ is the average number of particles per unit volume, $V$ is the volume occupied by the cloud, $\langle\mathbf{K}(\hat{\mathbf{q}})\rangle_{\xi}$ is the $4 \times 4$ single-particle extinction matrix averaged over all $M$ particles, and $\left\langle\mathbf{Z}\left(\hat{\mathbf{q}}, \hat{\mathbf{q}}^{\prime}\right)\right\rangle$ is the $4 \times 4$ single-particle phase matrix, also averaged over all particles [20].
A companion result of the microphysical theory of radiative transfer [22] allows one to compute the timeaveraged Poynting vector at the observation point $\mathbf{r}$ by integrating the product $\hat{\mathbf{q}} \tilde{I}(\mathbf{r}, \hat{\mathbf{q}})$ over all directions of the unit vector $\hat{\mathbf{q}}$ :

$$
\langle\mathbf{S}(\mathbf{r}, t)\rangle=\int_{4 \pi} \mathrm{d} \hat{\mathbf{q}} \hat{\mathbf{q}} \tilde{I}(\mathbf{r}, \hat{\mathbf{q}}) .
$$

Eqs. (24)-(26) are quite useful in practice. Firstly, Eq. (24) implies that if the underlying assumptions about the particulate medium are valid then a WCR such as that shown in Fig. 10 measures the quantity $\tilde{I}(\mathbf{r}, \hat{\mathbf{q}})$ provided that the reading of the WCR is averaged over a sufficiently long period of time. Therefore, by sampling a sufficiently dense grid of incoming directions $\hat{\mathbf{q}}$, one can determine the local time-averaged Poynting vector according to Eq. (26) and thereby solve the radiation-budget problem experimentally by using Eq. (1). Alternatively, by solving Eq. (25) and substituting the result in Eq. (26), one can solve the radiation-budget problem theoretically.

Secondly, the angular dependence of the measured function $\tilde{I}(\mathbf{r}, \hat{\mathbf{q}})$ can be analyzed by solving Eq. (25) for a representative range of physical models of the cloud and thereby retrieve valuable macro- and microphysical information about the particulate object. Furthermore, since the WCR selects only locally plane wavefronts propagating in directions very close to $\hat{\mathbf{q}}$, it is straightforward to convert the WCR into a directional photopolarimeter capable of measuring the entire column vector $\tilde{\mathbf{I}}(\mathbf{r}, \hat{\mathbf{q}})$. This measurement has been shown to contain additional implicit information about particle microphysics which can often be retrieved since solving Eq. (25) yields all four elements of $\tilde{\mathbf{I}}(\mathbf{r}, \hat{\mathbf{q}})$ at once (see, e.g., Refs. [28-31] and references therein).

\section{Panoramic radiometers}

The WCR depicted schematically in Fig. $5 b$ is an inherently monodirectional instrument. The basic optical scheme of a multidirectional panoramic radiometer is shown in Fig. 5e. In this case the role of the diaphragm is played by each individual pixel of the $L$-pixel CCD. For example, the pink wavefront propagating along the optical axis in the direction $\hat{\mathbf{q}}$ is detected by the central pixel of the $C C D$, while the blue wavefront propagating in the direction $\hat{\mathbf{q}}^{\prime}$ is not annihilated (cf. Fig. 5b) but rather is detected by an off-center pixel. In other words, each pixel is expected to measure the cumulative Poynting vector of the superposition of plane or near-plane wavefronts propagating in the direction defined by the corresponding \{objective lens, pixel\} filter. As a result, a single exposure of the CCD yields $L$ simultaneous measurements which can be thought of as forming a two-dimensional image.

For a host of technical reasons not discussed here specifically, accurate radiometric measurements with CCDs are more problematic than those with photomultipliers and photodiodes. However, the following note is of a more basic nature. As was discussed in Section 2, the quantum theory of the photoelectric effect is well established in the case of photodetection of a plane electromagnetic wave or a parallel polychromatic beam. 
The main practical result of this theory is summarized by Eq. (2) and helps making the measurement with a WCR a well-characterized and interpretable procedure. It also further illustrates the essential role played by the relay lens in Fig. 5b.

Unlike the optical scheme of a WCR shown in Fig. 5b, that of a panoramic radiometer shown in Fig. 5e implies that each pixel is located at the focal point of a converging spherical wavefront rather than being exposed to a plane wavefront. To the best of the author's knowledge, the quantum theory of photodetection of a converging spherical wave has not been developed yet. As a consequence, the quantitative relationship between the individual intensities of the plane wavefronts in Fig. 5e and the signals generated by the respective CCD pixels remains uncertain. This obviously makes theoretical interpretations of measurements with panoramic radiometers more uncertain than those with WCRs.

\section{Concluding remarks}

The issue of directional flow of electromagnetic energy is a very complex one. We have already indicated that the Poynting vector cannot be interpreted as characterizing the direction and rate of the local electromagnetic energy flow. This is especially true of a volume element $\Delta V$ containing absorbing matter since then the Poynting theorem (1) implies infinite energy accumulation inside $\Delta V$. In fact, this example, illustrates a major weakness of classical macroscopic electromagnetics which describes only linear interactions of the electromagnetic field with matter. This is acceptable in the case of nonabsorbing dielectrics since they are not modified by the field, but causes problems in the case of absorbing dielectrics. It is therefore not surprising that microscopic quantization of the electromagnetic field in absorbing dielectrics requires linking the dielectric to a thermal field reservoir [32-34]. The heuristic way to circumvent this issue in classical macroscopic electromagnetics is to postulate that if $W_{\Delta S}>0$ in Eq. (1) then the excess electromagnetic energy is transformed into other forms of energy via physical mechanisms not encompassed explicitly by the macroscopic Maxwell equations.

It is this heuristic patch that is at the very heart of applying classical macroscopic electromagnetics to the solution of radiation-budget problems. In particular, a disperse medium is called conservatively scattering if $W_{\Delta S}=0$ in Eq. (1). If, however, the application of Eq. (21) or Eqs. (25), (26), and (1) yields $W_{\Delta S}>0$ then it is usually claimed that the excess electromagnetic energy is converted into heat.

The complete modern description of the interaction of the electromagnetic field and matter is provided by QED. It is imperative to recognize that in general, the Hamiltonian describing the field-matter interaction is not controlled by the Poynting vector of the electromagnetic field but rather is defined in terms of the electric and magnetic field vectors. This factor has two important implications: (i) Eq. (2) has a rather narrow range of applicability; and (ii) although it is the intensity of the incident parallel beam that enters Eq. (2), it appears in the form of an average over a sufficiently long period of time. As a consequence, a photoelectric detector cannot measure the instantaneous local Poynting vector under any circumstances.

Despite this profound limitation, making a photoelectric detector the end component of the optical tract of a WCR can serve a useful purpose. Indeed, we have seen that if one makes far-field measurements described in Section 5 or places the WCR inside a very large random cloud composed of sparsely distributed particles then the time-averaged signal generated by the WCR can be modeled theoretically by solving the macroscopic Maxwell equations directly or by using a corollary of the Maxwell equations in the form of Eqs. (24) and (25). As a consequence, the WCR can be made a legitimate part of optical-characterization and radiationbudget applications.

This does not mean, however, that a WCR can be considered a universal quantifier of directional electromagnetic energy flow. The physical nature of the specific measurement afforded by a WCR depends on the type of the ambient electromagnetic field and thus on the object causing the field. Unless the actual functionality of the WCR is rigorously defined, endowing it with desirable rather than real measurement characteristics can be thoroughly misleading and can result in significant errors.

Our final remark concerns Eq. (25). One might claim that this is the standard radiative transfer equation postulated in the phenomenological radiative transfer theory based on vague energy-conservation and directionalenergy-propagation arguments. Furthermore, one might attribute to $\tilde{I}(\mathbf{r}, \hat{\mathbf{q}})$ primordial physical significance as the quantity specifying the angular distribution of electromagnetic energy flow at the point $\mathbf{r}$ over all propagation directions $\hat{\mathbf{q}} \in 4 \pi$.

The recently developed microphysical approach to radiative transfer $[20,22]$ shows that this interpretation of Eq. (25) and the quantity $\tilde{I}(\mathbf{r}, \hat{\mathbf{q}})$, traditionally called the specific intensity, is thoroughly incorrect [7]. The quantity $\tilde{I}(\mathbf{r}, \hat{\mathbf{q}})$ does enter the formula (26) for the time-averaged Poynting vector. However, we have already seen that even the Poynting vector cannot be legitimately claimed to specify the direction of time-averaged electromagnetic energy flow, and so there is even less justification for ascribing any "directional energy" content to the specific intensity. The quantity $\tilde{I}(\mathbf{r}, \hat{\mathbf{q}})$ is nothing but a formal solution of the intermediate Eq. (25) and appears as a byproduct of the purely mathematical derivation of Eqs. (24) and (26) from the macroscopic Maxwell equations.

To summarize the above discussion, there has been a paradigm-changing shift in the physical understanding of the discipline of directional photometry established phenomenologically 250 years ago by Bouguer and Lambert. From allegedly describing the "directional flow of light" (or, more generally, electromagnetic energy), photometry has been reduced to making measurements with WCRs and modeling these measurements theoretically on the basis of fundamental physical theories. In the words of Roy Glauber, "A photon is what a photodetector detects" [35]. Paraphrasing Glauber, we can say that the specific intensity is what a WCR measures, provided that the parameters of the particulate medium in question are consistent with the assumptions used in the derivation of Eqs. (24)-(26). There 
is still an urgent need to understand the practical functionality of a WCR in more complex situations not discussed in this paper.

The physical theory of measurements with panoramic radiometers needs further refinement. Needless to say, a useful byproduct of this refinement would be a better understanding of the optical functionality of the most important panoramic radiometer called the human eye.

\section{Acknowledgments}

I appreciate numerous illuminating discussions with Matthew Berg, Brian Cairns, Michael Kahnert, and Daniel Mackowski as well as helpful comments by two anonymous referees. This research was funded by the NASA Remote Sensing Theory Program managed by Lucia Tsaoussi and the NASA Radiation Sciences Program managed by Hal Maring.

\section{References}

[1] Stratton JA. Electromagnetic theory. New York: McGraw Hill; 1941.

[2] Bukshtab M. Applied photometry, radiometry, and measurements of optical losses. Dordrecht: Springer; 2012.

[3] Bouguer P. Optical treatise on the gradation of light. Toronto: University of Toronto Press; 1961 [English translation by WEK Middleton of the French original published in 1760].

[4] Lambert JH. Photometry, or on the measure and gradations of light, colors and shade. New York: Illuminating Engineering Society of North America; 2001 [English translation by DL DiLaura of the Latin original published in 1760].

[5] Mishchenko MI. Gustav Mie and the fundamental concept of electromagnetic scattering by particles: a perspective. J Quant Spectrosc Radiat Transfer 2009;110:1210-22.

[6] Mishchenko MI, Tishkovets VP, Travis LD, Cairns B, Dlugach JM, Liu L, et al. Electromagnetic scattering by a morphologically complex object: fundamental concepts and common misconceptions. J Quant Spectrosc Radiat Transfer 2011;112:671-92.

[7] Mishchenko MI. Directional radiometry and radiative transfer: a new paradigm. J Quant Spectrosc Radiat Transfer 2011;112: 2079-94.

[8] Jackson JD. Classical electrodynamics. New York: Wiley; 1999.

[9] Hertz H. Ueber einen Einfluss des ultravioletten Lichtes auf die electrische Entladung. Ann Phys 1887;267:983-1000.

[10] Dodd JN. Atoms and light: interactions. New York: Plenum Press; 1991.

[11] Mandel L, Wolf E. Optical coherence and quantum optics. Cambridge, UK: Cambridge University Press; 1995.

[12] Stoletov AG. Actinoelectric investigations. St. Petersburg: V. Demakov Publishing House; 1889. 〈http://e-heritage.ru/ras/view/publi cation/general.html?id $=42070560>$ [in Russian].

[13] Kimble HJ, Mandel L. Photoelectric detection of polychromatic light. Phys Rev A 1984;30:844-50.
[14] Lee CM. Spin polarization and angular distribution of photoelectrons in the Jacob-Wick helicity formalism. Application to autoionization resonances. Phys Rev A 1974;10:1598-604.

[15] Huang K-N. Theory of angular distribution and spin polarization of photoelectrons. Phys Rev A 1980;22:223-39.

[16] Berglund CN, Spicer WE. Photoemission studies of copper and silver: theory. Phys Rev 1964;136:A1030-44.

[17] Munz P. Angular dependence and vectorial effect of the photoemission from EuO. Solid State Commun 1975;17:627-30.

[18] Chyba TH, Mandel L. Angular sensitivity of a vacuum photodiode, or does a photodetector always count absorbed photons? J Opt Soc Am B 1988;5:1305-11.

[19] Mishchenko MI, Travis LD, Lacis AA. Scattering, absorption, and emission of light by small particles. Cambridge: Cambridge University Press; 2002. 〈http://www.giss.nasa.gov/ crmim/ books.html $>$.

[20] Mishchenko MI, Travis LD, Lacis AA. Multiple scattering of light by particles: radiative transfer and coherent backscattering. Cambridge: Cambridge University Press; 2006. 〈http://www.giss.nasa. gov/staff/mmishchenko/publications/Book3.pdf $\rangle$.

[21] Goodman JW. Introduction to Fourier optics. Englewood (CO): Roberts \& Company; 2005.

[22] Mishchenko MI. The Poynting-Stokes tensor and radiative transfer in discrete random media: the microphysical paradigm. Opt Express 2010;18:19770-91.

[23] Novotny L, Hecht B. Principles of nano-optics. Cambridge (UK): Cambridge University Press; 2012.

[24] Bohren CF, Huffman DR. Absorption and scattering of light by small particles. New York: Wiley; 1983.

[25] Berg MJ, Sorensen CM, Chakrabarti A. Extinction and the optical theorem. I. Single particles. J Opt Soc Am A 2008;25:1504-13.

[26] Berg MJ, Sorensen CM, Chakrabarti A. Extinction and the optical theorem. II. Multiple particles. J Opt Soc Am A 2008;25:1514-20.

[27] Mishchenko MI, Berg MJ, Sorensen CM, van der Mee CVM. On definition and measurement of extinction cross section. J Quant Spectrosc Radiat Transfer 2009;110:323-7.

[28] Videen G, Yatskiv Ya, Mishchenko M, editors. Photopolarimetry in remote sensing. Dordrecht (The Netherlands): Kluwer; 2004.

[29] Hovenier JW, Van der Mee C, Domke H. Transfer of polarized light in planetary atmospheres-basic concepts and practical methods. Dordrecht (The Netherlands): Kluwer; 2004.

[30] Mishchenko MI, Rosenbush VK, Kiselev NN, Lupishko DF, Tishkovets VP, Kaydash VG, et al. Polarimetric remote sensing of Solar system objects. Kyiv: Akademperiodyka; 2010. http://arxiv.org/ abs/1010.1171.

[31] Mishchenko MI, Yatskiv YaS, Rosenbush VK, Videen G. Polarimetric detection, characterization, and remote sensing. Berlin: Springer; 2011.

[32] Huttner B, Barnett SM. Quantization of the electromagnetic field in dielectrics. Phys Rev A 1992;46:4306-22.

[33] Ho S-T, Kumar P. Quantum optics in a dielectric: macroscopic electromagnetic-field and medium operators for a linear dispersive lossy medium - a microscopic derivation of the operators and their commutation relations. J Opt Soc Am B 1993;10:1620-36.

[34] Suttorp LG, Wubs M. Field quantization in inhomogeneous absorptive dielectrics. Phys Rev A 2004;70:013816.

[35] Muthukrishnan A, Scully MO, Zubairy MS. The concept of the photon-revisited. In: Roychoudhuri C, Kracklauer AF, Creath K, editors. The nature of light: what is a photon? Boca Raton (FL): CRC Press; 2008. p. 37-57. 